\title{
Translational evidence for RRM2 as a prognostic biomarker and therapeutic target in Ewing sarcoma
}

\author{
Shunya Ohmura 1,2,3, Aruna Marchetto ${ }^{3}$, Martin F. Orth³ , Jing Li ${ }^{1,2}$, Susanne Jabar ${ }^{4,5}$, Andreas Ranft ${ }^{4,5}$, \\ Endrit Vinca ${ }^{1,2}$, Katharina Ceranski ${ }^{1,2}$, Martha J. Carreño-Gonzalez ${ }^{1,2}$, Laura Romero-Pérez ${ }^{1,2,3}$, \\ Fabienne S. Wehweck ${ }^{3}$, Julian Musa 1,2,3,6, Felix Bestvater ${ }^{7}$, Maximilian M. L. Knott ${ }^{3}$, Tilman L. B. Hölting ${ }^{3}$, \\ Wolfgang Hartmann ${ }^{8}$, Uta Dirksen ${ }^{4,5}$, Thomas Kirchner ${ }^{9,10}$, Florencia Cidre-Aranaz ${ }^{1,2,3}$ and \\ Thomas G. P. Grünewald ${ }^{1,2,3,11^{*}}$
}

Keywords: Ewing sarcoma, RRM2, Targeted therapy, Prognostic biomarker, Paediatric oncology, Triapine, Chemoresistance

\begin{abstract}
Main text
Ewing sarcoma (EwS) is an aggressive bone- or soft tissueassociated malignancy, characterised by the fusion oncoprotein EWSR1-FLI1 [1]. Over the past decades further therapeutic development for this devastating childhood tumour has remained relatively stagnant [2], especially for patients with metastatic or recurrent disease [3, 4]. To develop more effective and specific treatment options we investigated potential therapeutic targets by exploring putative downstream genes of EWSR1-FLI1.

We took advantage of publicly available 'omics' data and filtered them in a multi-step approach (Fig. 1a): First, we interrogated a gene expression dataset comprising 50 primary EwS and 929 samples from 71 normal tissue types to identify overexpressed genes (min. $\log 2$ fold increase =2) in EwS, which yielded 292 candidates (Fig. 1b, Supplementary Table 1). Second, we filtered for those genes whose overexpression was significantly negatively correlated with patients' overall survival in a dataset of matched gene expression and survival data of
\end{abstract}

\footnotetext{
*Correspondence: t.gruenewald@kitz-heidelberg.de

${ }^{2}$ Division of Translational Pediatric Sarcoma Research (B410), German

Cancer Research Center (DKFZ) \& Hopp-Children's Cancer Center (KiTZ), Im Neuenheimer Feld 280, 69210 Heidelberg, Germany

Full list of author information is available at the end of the article
}

166 EwS patients [5] that covered 280 of the 292 overexpressed genes (96\%) (Fig. 1c), identifying 22 candidates (Supplementary Table 1). Third, we focused on druggable targets possessing kinase or other enzymatic functions for which specific inhibitors and their pharmacokinetic data were available, but were still not (pre) clinically tested in EwS. This survey identified ribonucleotide reductase regulatory subunit M2 (RRM2) as the single putative target with a prominently negative association with patients' overall survival (Fig. 1d). The ribonucleotide reductase (RNR) catalyses the conversion of ribonucleoside diphosphates to deoxyribonucleoside diphosphates, the rate-limiting process for de novo deoxyribonucleoside triphosphates synthesis. RNR is composed of two subunits, ribonucleotide reductase catalytic subunit M1 (RRM1) and either RRM2 or ribonucleotide reductase regulatory TP53 inducible subunit M2 (RRM2B) [6]. Notably, RRM2B is neither overexpressed in EwS nor negatively correlates with patients' outcome (Supplementary Figs. 1a,b), and RRM1 is far less overexpressed in EwS compared to RRM2 (Supplementary Figs. 1a,b,c). Similar to primary EwS tumours, assessment of transcriptomes from 18 EwS cell line models (including A-673 and TC-71) also exhibited that, while $R R M 2$ and $R R M 1$ were similarly highly expressed in EwS original author(s) and the source, provide a link to the Creative Commons licence, and indicate if changes were made. The images or other third party material in this article are included in the article's Creative Commons licence, unless indicated otherwise in a credit line to the material. If material is not included in the article's Creative Commons licence and your intended use is not permitted by statutory regulation or exceeds the permitted use, you will need to obtain permission directly from the copyright holder. To view a copy of this licence, visit http://creativecommons.org/licenses/by/4.0/. The Creative Commons Public Domain Dedication waiver (http://creativeco mmons.org/publicdomain/zero/1.0/) applies to the data made available in this article, unless otherwise stated in a credit line to the data. 
cell line models, $R R M 2$ was on average $\sim$ ninefold higher expressed than $R R M 2 B(P<0.0001)$. These observations, together with the absence of a negative survival association of $R R M 2 B$ in EwS (Supplementary Fig. 1b), suggested that RRM2B, although being structurally similar to RRM2 [6], may play a subordinate role in EwS.

Prior reports suggested that RRM2 may contribute to the proliferative phenotype of EwS [7, 8]. However, its role in primary EwS tumours remains unclear. To gain first insights into the biological function of RRM2 in EwS, we carried out gene ontology (GO) enrichment analysis of RRM2 co-expressed genes in 166 EwS tumours, which revealed that high RRM2 expression is closely correlated with cell proliferation-associated gene signatures (Fig. 1e), suggesting that high RRM2 expression may contribute to an aggressive clinical course by promoting tumour growth. Next, we analysed the potential association between RRM2 protein levels, clinicopathological prognostic factors, and clinical outcomes in tissue microarrays (TMA) from EwS tumours of 122 patients (Supplementary Table 2, Supplementary Fig. 2a). In agreement with the findings at the mRNA level (Fig. 1d), high RRM2 protein expression was significantly $(P=0.0095)$ associated with poor overall survival (Fig. 1f). Correspondence analyses of individual cohorts and the joint-cohort (after exclusion of 6 samples (3.6\%) from the mRNA-cohort that were in overlap with the TMA cohort) revealed that high RRM2 expression was significantly associated with metastatic disease at diagnosis $(P=0.0004)$ and occurrence of metastatic and/or local relapse $(P=0.0095$; only available for the TMA cohort) (Supplementary Table 3), supporting that high RRM2 expression promotes an aggressive phenotype. Conversely, RRM2 inhibition by doxycycline (Dox)-inducible shRNA-mediated gene silencing inhibited proliferation and clonogenic growth of three EwS cell lines, and induced cell death in vitro (Supplementary Figs. 2b,c). Consistent with these functional experiments, transcriptome profiling upon $R R M 2$ silencing in two EwS cells demonstrated downregulation of cell cycle and proliferation-associated gene signatures (Supplementary Fig. 2d). Similarly, RRM2 knockdown significantly reduced tumour growth of two xenografted EwS cells (Figs. 1g,h). This antineoplastic effect was accompanied by increased apoptosis and DNA damage, as assessed by immunohistochemistry for cleaved caspase 3 (CC3) and $\gamma$ H2A.X, respectively (Fig. 1i, Supplementary Fig. 2e).

Generally, the activity of RNR can be blocked by irreversible RRM1 inhibition using gemcitabine, or by RRM2-specific inhibitors such as hydroxyurea or the more potent compound triapine (alias 3-AP) [6, 9]. Although gemcitabine is used for palliative treatment of EwS patients, EwS tumours rapidly develop a relative resistance [10]. Consistently, we found that long-term treatment of EwS cells with ascending doses of either doxorubicin (A-673, ES7, EW-7, TC-71), gemcitabine (A-673, ES7, TC-71) or triapine (A-673) led to acquisition of relative resistance phenotypes in vitro (Supplementary Fig. 3a), where we noted a relatively fast and strong increase of the relative resistance towards gemcitabine ( $>2,000$-fold increase in $\mathrm{IC}_{50}$ within $\sim 6$ weeks), compared doxorubicin ( $\sim$ fourfold increase in $\sim 28$ weeks) and triapine $(\sim$ sevenfold increase in $\sim 20$ weeks) (Supplementary Fig. 3b), further suggesting that gemcitabine has limited potential for clinical treatment with curative intent. Thus, we focused on triapine for further functional analyses. First, to assess functional dependency of triapine on RRM2 expression, we performed drugresponse assays using triapine in EwS cells with/without $R R M 2$ silencing, which demonstrated that knockdown of $R R M 2$ led to a $\sim$ twofold decrease of the IC50 for triapine in A-673 EwS cells, indicating that higher doses of the drug are required to fully block RRM2 activity in case of high RRM2 expression (Supplementary. Figure 3c). Such

(See figure on next page.)

Fig. 1 RRM2 is highly overexpressed in EwS, correlates with poor patient outcome, and constitutes a putative therapeutic target. a Schematic description of the filtering process for identification of therapeutically relevant target candidates. $\mathbf{b}$ Analysis of RRM2 mRNA expression levels in 50 EwS primary tumours compared to 929 normal tissues samples from 71 tissue types. Data are shown as log2 fold increase normalized to expression values of normal tissues. The dotted line indicates the cut-off value of 2 for candidate selection. c Analysis of overall survival time of 166 EwS patients stratified for candidate gene expression. P-values $(-\log 10)$ were determined in Kaplan-Meier analyses using a Mantel-Haenszel test (Bonferroni-adjusted for multiple testing). The dotted line indicates a significance value of 1.3. d Kaplan-Meier survival analysis of 166 EwS patients stratified by the $78^{\text {th }}$ percentile RRM2 expression. $P$-value determined by log-rank test. $\mathbf{e}$ Left: Heat map for gene expression which is positively or negatively correlated with RRM2 expression in 166 EwS. Right: Gene ontology (GO) enrichment analysis of RRM2 and its co-expressed genes derived from gene expression data sets of 166 EwS tumours. Pearson correlation coefficients between RRM2 and other genes were determined, of which those with $\left|r_{\text {pearson }}\right|>0.5$ were further analysed by GO enrichment analysis. $\mathbf{f}$ Kaplan-Meier survival analysis of 122 EwS patients stratified by RRM2 protein expression (low IRS $\leq 2$, high IRS $>2$ ). P-values were determined by log-rank test. $\mathbf{g}$ Analysis of tumour growth of EwS cell lines A-673 and TC-71 harbouring Dox-inducible shRRM2 constructs or non-targeting shRNA (shControl) xenografted in NSG mice. Once tumours were palpable, animals were randomized in Dox (+) or Dox (-) group. Tumour growth on time course and $\mathbf{h}$ Tumour weight at the experimental endpoint. Arrows indicate treatment start. Values were normalized to Dox (-). Horizontal bars represent means and whiskers SEM. FC, fold change. $P$-values were calculated at the experimental endpoint with two-sided (tumour growth) or one-sided (tumour weight) Mann-Whitney test. i Quantification of positive cells for cleaved caspase-3 (CC3) (left) and YH2A.X (right). Values were normalized to Dox (-). Horizontal bars represent means and whiskers SEM. FC, fold change. P-values were calculated at the experimental endpoint using a two-sided Mann-Whitney test 
a

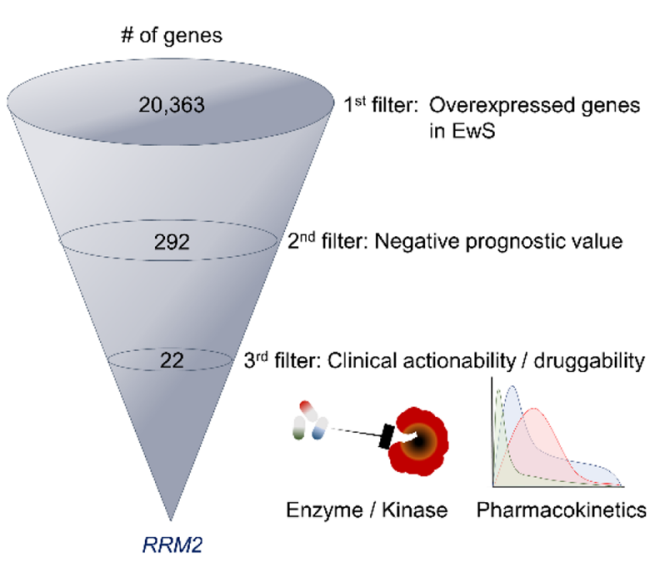

e Low $\overline{166}_{\mathrm{EwS}}$ High

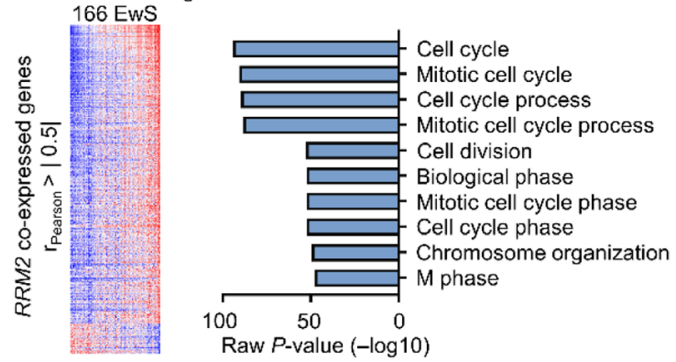

g

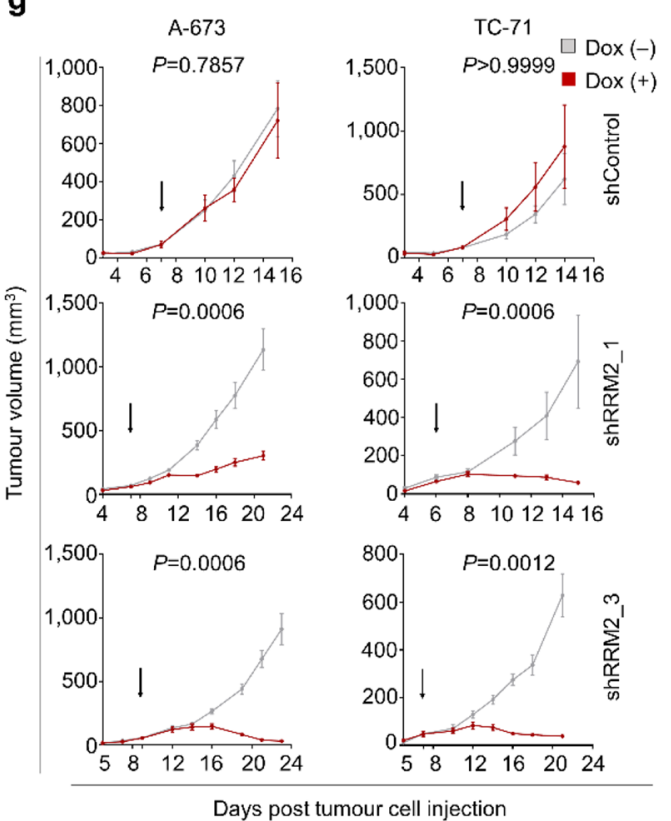

b

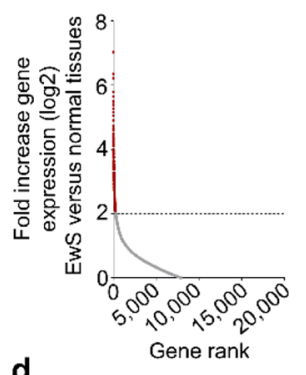

c

d

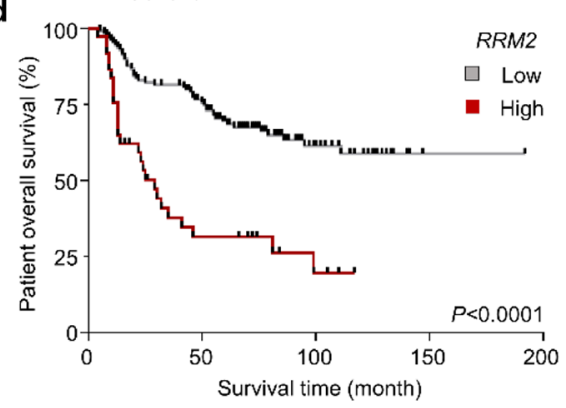

f

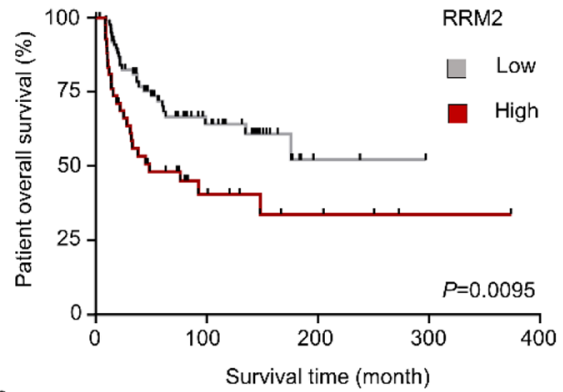

h

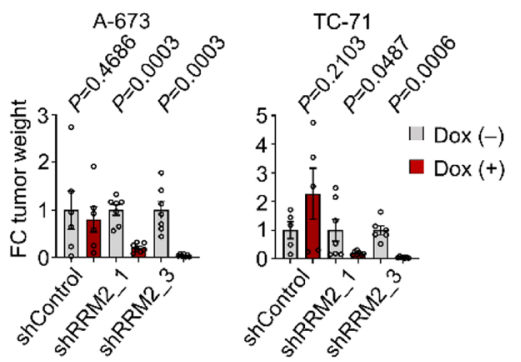

i

Cleaved caspase-3

A-673

TC-71

A-673 ${ }^{\text {YH2A.X }}$ TC-71

$\frac{\infty}{\bar{\alpha}} 6$

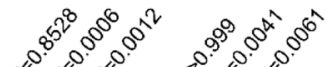

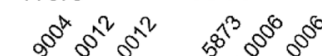

$\frac{\infty}{\overline{0}} 10$

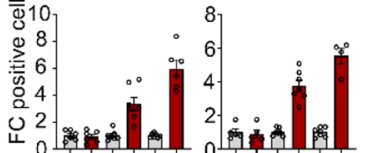

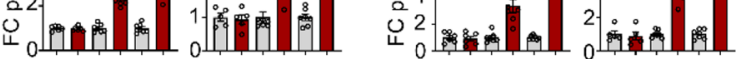
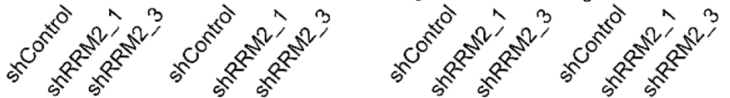

Fig. 1 (See legend on previous page.) 
differential effect on sensitivity towards triapine was not observed in A-637 cells expressing a non-targeting control shRNA. Moreover, we observed an $\sim$ twofold increase of RRM2 expression in triapine-resistant A-673 (A-673/ TR) compared to parental A-673 EwS cells, suggesting that $R R M 2$ upregulation can be a potential mechanism for acquiring triapine-resistance in A-673 EwS cells (Supplementary Fig. 3d). Dose-response assays revealed that EwS cells were very sensitive towards triapine compared to osteosarcoma cells and non-transformed EwS patientderived mesenchymal stem cells (mean $\mathrm{IC}_{50}$ values 0.35 , 1.63, $101.63 \mu \mathrm{M}$, respectively) (Fig. 2a). Likewise, triapine treatment significantly reduced clonogenic growth of EwS cells at clinically relevant doses [11, 12] (Fig. 2b). Interestingly, doxorubicin or gemcitabine resistant EwS cells (designated EwS/DR or EwS/GR, respectively) still retained triapine sensitivity (Fig. 2c), suggesting therapeutic potential of triapine for EwS refractory towards conventional chemotherapy. Strikingly, we could confirm a significant reduction of tumour growth by triapine treatment compared to controls in a subcutaneous xenograft model (Fig. 2d). Although triapine treatment was accompanied by weight loss (on average $~ 5 \%$ at the experimental endpoint) (Supplementary Fig. 3e), no morphological changes of inner organs were observed including the gastrointestinal tract as assessed by histological analysis (Supplementary Fig. 3f). Although it is interesting to note that EwS cells resistant to gemcitabine, which covalently binds and thus inactivates RRM1 [13], still retain sensitivity towards triapine (Fig. 2c), it should be noted that triapine may not be entirely specific for RRM2. Triapine presumably disrupts a tyrosyl free radical by labilising di-iron molecules on the small subunits of ribonucleotide reductase $[6,14]$. This proposed mechanism of action for triapine can clinically manifest as a reversible adverse effect such as methemoglobinemia, which is probably caused by the iron chelating effect of triapine, interrupting recovery cycles from methaemogloblin to haemoglobin [15]. To mitigate this toxicity, the small molecule $\mathrm{COH} 29$ has been developed that, upon binding to RRM2 subunits, interferes with the molecular interface of RRM1 and RRM2 subunits and thus inhibits its reductase function [14]. Yet, its clinical efficacy and safety remain to be investigated. Another approach for more specific RRM2 inhibition has been undertaken with antisense oligonucleotide-based techniques, exemplified by therapeutic silencing of RRM 2 by GTI-2040, which, however, showed little clinical benefit in several clinical trials [16-18]. Hence, despite our data strongly support RRM2 as an actionable and valuable drug target in EwS, and triapine as a potential lead candidate drug for preferential RRM2 inhibition, the development of even more specific RRM2 inhibitors is desirable.

We next explored effective drug combinations with triapine. Based on known functions of RRM2 in DNA synthesis and DNA repair [6] we examined combinatory applications of triapine with standard chemotherapeutics, doxorubicin, etoposide or vincristine, or poly ADP-ribose polymerase (PARP) inhibitors. Unexpectedly, we observed rather antagonistic effects (Supplementary Figure $4 \mathrm{a}$ ). To identify rational combinations, we analysed integrated transcriptome profiles of two EwS cells upon RRM2 silencing and triapine treatment, revealing 263 commonly up- and downregulated genes (Supplementary Table 4). GO enrichment analysis demonstrated significant enrichment for cell cycleassociated processes, especially regulation of mitotic cell cycle-associated genes (Fig. 2e), which is consistent with the observation that RRM2 inhibition caused G1/S-phase cell cycle arrest $[19,20]$. Thus, we reasoned that RRM2 may synergise with checkpoint inhibitors targeting CHEK1 (checkpoint kinase 1) or WEE1 (WEE1 G2 checkpoint kinase), which were highly significantly $(P<0.0001)$ co-expressed with $R R M 2$ in 166

\footnotetext{
(See figure on next page.)

Fig. 2 Triapine inhibits EwS growth in vitro and in vivo and synergise with cell cycle checkpoint inhibitors in vitro. a Dose-response analysis of triapine in EwS, osteosarcoma and mesenchymal stem cells. b Analysis of clonogenic growth of A-673, TC-71, and EW-7 EwS cells upon triapine treatment. c Left: Dose-response analysis of triapine in chemoresistant EwS cells (A-673/DR or A-673/GR). Right: magnitudes of doxorubicin or gemcitabine resistance shown by fold increase in $\mathrm{IC}_{50}$ compared to those of parental cells. $\mathbf{d}$ Analysis of tumour growth upon triapine treatment in A-673 cell line in vivo. Images of resected xenografts (upper), tumour growth (lower left), and tumour weight at the experimental endpoint (lower right) of NSG mice xenografted with A-673 EwS cells upon treatment with triapine. Once tumours reached $5 \mathrm{~mm}$ in average diameter, animals were randomized in treatment group (30 mg/kg i.p.) or control group (DMSO) $(n=8$ animals per group). The arrow indicates treatment start. Horizontal bars represent means and whiskers SEM. P-values were calculated at the experimental endpoint with two-sided (tumour growth) or one-sided (tumour weight) Mann-Whitney test. e Integrative Gene Ontology (GO) enrichment analysis of gene expression microarray data generated in A-673 and ES7 cells after RRM2 silencing or pharmacological RRM2 inhibition by triapine (corresponding IC 50 of $0.44 \mu \mathrm{M}$ or $0.65 \mu \mathrm{M}$, respectively). f Correlation of gene expression between RRM2 and CHEK1 or WEE1 in 166 EwS. Each dot represents an individual expression value. Solid red lines indicate a trend line created by a simple linear regression. P-values were calculated by a two-tailed t-test. $\mathbf{g}$ Drug interaction and combination efficiency analysis between triapine and CHEK1 inhibitor (CCT245737) or WEE1 inhibitor (MK-1775) in 4 EwS cell lines (A-673, ES7, EW-7, TC-71) assessed by combination index. Cl value $<1$ indicative of synergistic, $\mathrm{Cl}=1$ additive, and $\mathrm{Cl}>1$ antagonistic $\mathbf{h}$ Drug interaction and combination efficiency estimation between triapine and CHEK1 inhibitor (CCT245737) or WEE1 inhibitor (MK-1775) in A-673 EwS cell line assessed by SynergyFinder 2.0. ZIP synergy score > 10, likely to be synergistic; between -10 and 10, likely to be additive; <-10, likely to be antagonistic
} 


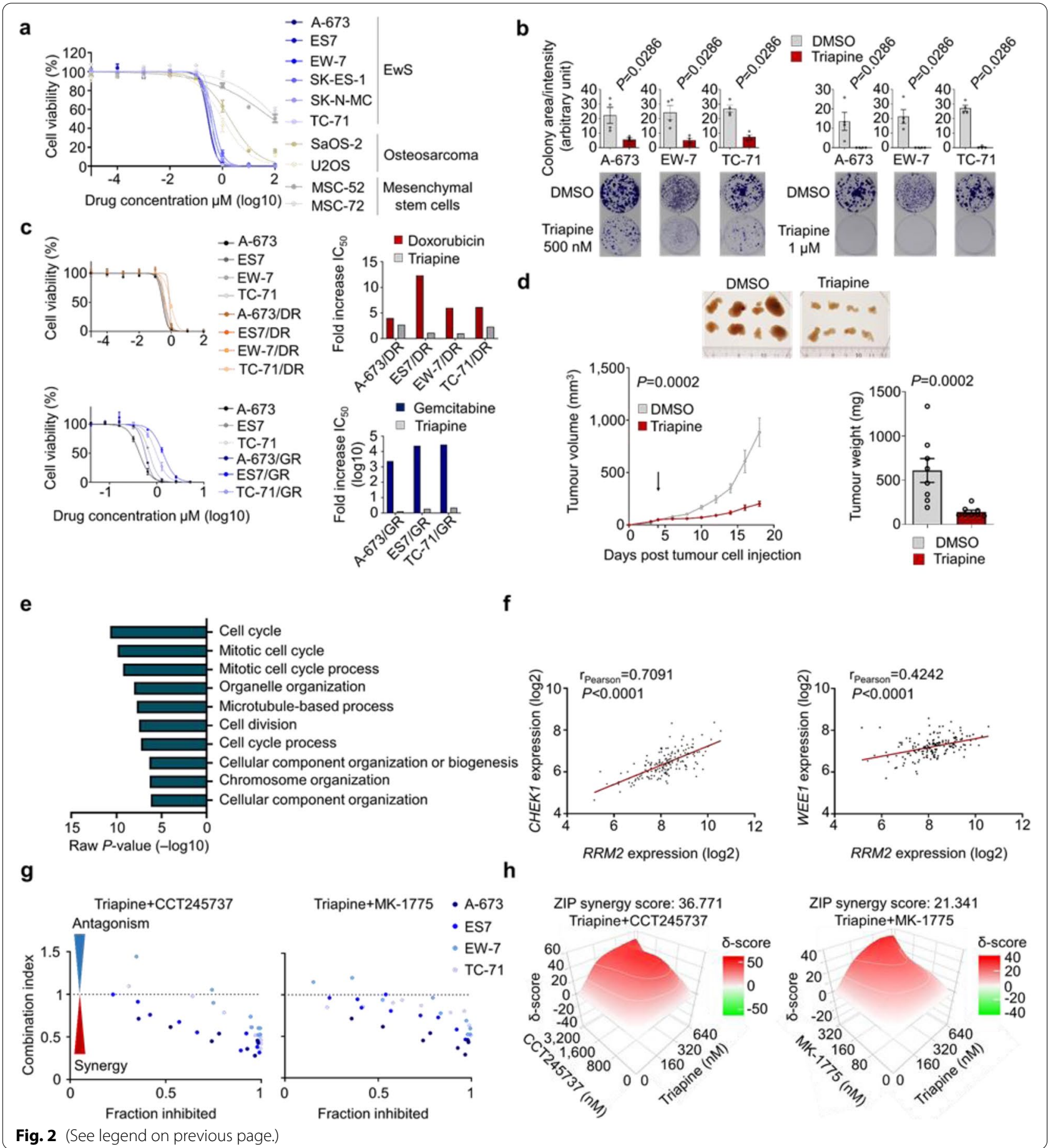

EwS tumours (Fig. 2f). In drug combination assays we observed a strong synergism between triapine and a CHEK1 inhibitor (CCT245737) or a WEE1 inhibitor (MK-1775) across four EwS cells (Fig. 2g,h, Supplementary Fig. 4b). Overall, these results provide a rationale for therapeutic combination of triapine with cell cycle checkpoint inhibitors. A recent study pointed out that the drug combination of hydroxyurea and a CHEK1 inhibitor (GDC-0575) can circumvent toxicities caused by the combination of gemcitabine and GDC-0575, which may imply a more manageable combinatory application through RRM2 inhibition and CHEK1 inhibitors [21]. 


\section{Conclusions}

Collectively, our results establish RRM2 as a promising actionable therapeutic target for EwS, even in chemotherapy-refractory cases, and suggest that the combination of triapine with cell cycle checkpoint inhibitors may be highly effective. Moreover, our integrative study of two independent cohorts provides evidence for RRM2 as novel and robust prognostic biomarker that can be readily assessed by immunohistochemistry in routine diagnostics. Thus, our findings may have immediate translational relevance for patients affected by this devastating disease.

\begin{abstract}
Abbreviations
3-AP: 3-Aminopyridine-2-carboxaldehyde thiosemicarbazone; CC3: Cleaved caspase-3; CHEK1: Checkpoint kinase 1; Cl: Combination index; Dox: Doxycycline; DR: Doxorubicin-resistant; EwS: Ewing sarcoma; EWSR1: Ewing sarcoma breakpoint region 1; FFPE: Formalin-fixed and paraffin-embedded; FLI1: Friend leukaemia virus integration 1; GO: Gene ontology; GR: Gemcitabine-resistant; GSEA: Gene set enrichment analysis; IHC: Immunohistochemistry; IRS: Immune reactive score; MSC: Mesenchymal stem cell; NSG: NOD/SCID/gamma; PARP: Poly ADP-ribose polymerase; RNR: Ribonucleotide reductase; RRM1: Ribonucleotide reductase catalytic subunit M1; RRM2: Ribonucleotide reductase regulatory subunit M2; RRM2B: Ribonucleotide reductase regulatory TP53 inducible subunit M2; TMA: Tissue microarray; TR: Triapine-resistant; WEE1: WEE1 G2 checkpoint kinase; WGCNA: Weighted gene co-expression network analysis; ZIP: Zero Interaction Potency.
\end{abstract}

\section{Supplementary Information}

The online version contains supplementary material available at https://doi. org/10.1186/s12943-021-01393-9.

\section{Additional file 1. Methods.}

Additional file 2: Supplementary Figure 1. $R R M 1, R R M 2$, and $R R M 2 B$ expression in EwS tumours, normal tissues and EWS cell line models, and their association with overall survival in 166 EwS patients. Supplementary Figure 2. RRM2 silencing inhibits cell proliferation and clonogenic growth in EwS in vitro, and representative immunohistochemical staining. Supplementary Figure 3. Development of chemoresistance in EwS cell lines, functional dependency of triapine on RRM2, and adverse effects of triapine treatment in vivo. Supplementary Figure 4. Drug interaction and combination efficiency of triapine with chemotherapeutics, PARP inhibitors, CHEK1 inhibitor or WEE1 inhibitor.

Additional file 3: Supplementary Table 1. Overexpressed genes in EwS compared to normal tissues and their prognostic relevance. Supplementary Table 2. Clinicopathological characteristics of EwS patients for the mRNA (discovery) and TMA-cohort (validation). Supplementary Table 3. Multivariate analysis for RRM2 expression and clinical parameters in the mRNA (discovery), TMA-cohort (validation) and joint-cohort. Supplementary Table 4. Commonly regulated genes upon RRM2 silencing and pharmacological inhibition by triapine. Supplementary Table $\mathbf{5}$. Oligonucleotide sequences.

\section{Acknowledgements}

We are grateful to M. Melz for constructing TMAs, to S. Stein and F. Zahnow for experimental assistance, and to A. Heier and A. Sendelhofert for technical assistance in establishing immunohistochemical staining procedures.

\section{Authors' contributions}

S.O. and T.G.P.G. conceived the study, wrote the manuscript and designed the figures and tables. S.O., A.M., and E.V. performed in vitro experiments. S.O. performed in vivo experiments, and F.C.A. helped in coordination of in vivo experiments. S.O., M.F.O., K.C., M.J.C.G., F.C.A., J.L., and T.G.P.G. performed bioinformatic and statistical analyses. S.O. and F.W. scored tissue-microarrays. S.J., A.R., U.D., and W.H. provided gene expression data, tissue-microarrays and helped in statistical analysis of clinical data. M.F.O., J.L., F.C.A., L.R.P., M.M.L.K., T.L.B.H., J.M. contributed to experimental procedures. F.B. and T.K. provided laboratory infra-structure and histological guidance. T.G.P.G. supervised the study and data analysis. All authors read and approved the final manuscript.

\section{Authors' information}

Not applicable.

\section{Funding}

This work was mainly supported by a grant from the Deutsche Forschungsgemeinschaft (DFG-391665916 to S.O.). In addition, the laboratory of T.G.P.G. is supported the Matthias-Lackas Foundation, the Dr. Leopold and Carmen Ellinger Foundation, the Dr. Rolf M. Schwiete Foundation, the Boehringer-Ingelheim Foundation, the German Cancer Aid (DKH-70112257; DKH-70114111), the Gert and Susanna Mayer Foundation, the SMARCB1association, and the Barbara and Wilfried Mohr foundation. The working group of U.D. (S.J. and A.R) is supported by the German Cancer Aid (DKH-70113419, DKH-70112418), the Gert and Susanna Mayer Foundation, and the Barbara and Hubertus Trettner Foundation. T.L.B.H. was supported by a Mildred-Scheel scholarship from the German Cancer Aid.

\section{Availability of data and materials}

Original microarray data used in this study were deposited at the National Centre for Biotechnology Information (NCBI) GEO under accession numbers GSE166415 and GSE166419. Custom code is available from the corresponding author upon reasonable request.

\section{Declarations}

\section{Ethics approval and consent to participate}

Human tissue samples were retrieved from the tissue archives of the Institute of Pathology of the LMU Munich (Germany) or the Gerhard-Domagk Institute of Pathology of the University of Münster (Germany) upon approval of the institutional review board. All patients provided informed consent. Tissuemicroarrays (TMAs) were stained and analysed with approval of the ethics committee of the LMU Munich (approval no. 550-16 UE). Animal experiments were approved by the local authorities and performed in accordance with ARRIVE guidelines, recommendations of the European Community (86/609/ EEC), and United Kingdom Coordinating Committee on Cancer Research (UKCCCR) guidelines for the welfare and use of animals in cancer research.

\section{Consent for publication}

Not applicable.

\section{Competing interests}

The authors declare no conflict of interest.

\section{Author details}

${ }^{1}$ Hopp Children's Cancer Center Heidelberg (KiTZ), Heidelberg, Germany. ${ }^{2}$ Division of Translational Pediatric Sarcoma Research (B410), German Cancer Research Center (DKFZ) \& Hopp-Children's Cancer Center (KiTZ), Im Neuenheimer Feld 280, 69210 Heidelberg, Germany. ${ }^{3}$ Max-Eder Research Group for Pediatric Sarcoma Biology, Institute of Pathology, Faculty of Medicine, LMU Munich, Munich, Germany. ${ }^{4}$ Pediatrics III, West German Cancer Centre, University Hospital Essen, Essen, Germany. ${ }^{5}$ German Cancer Consortium (DKTK), partner site Essen, Essen, Germany. ${ }^{6}$ Department of General, Visceral and Transplantation Surgery, Heidelberg University Hospital, Heidelberg, Germany. ${ }^{7}$ Light Microscopy Facility, German Cancer Research Center (DKFZ), German Cancer Consortium (DKTK), Heidelberg, Germany. ${ }^{8}$ Division of Translational Pathology, Gerhard-Domagk-Institute for Pathology, University Hospital Münster, Münster, Germany. ${ }^{9}$ Institute of Pathology, Faculty of Medicine, LMU Munich, Munich, Germany. ${ }^{10}$ German Cancer Consortium (DKTK), partner site Munich, Munich, Germany. ${ }^{11}$ Institute of Pathology, Heidelberg University Hospital, Heidelberg, Germany.

Received: 19 March 2021 Accepted: 12 July 2021

Published online: 27 July 2021 


\section{References}

1. Grünewald TGP, Cidre-Aranaz F, Surdez D, Tomazou EM, de Álava E, Kovar H, et al. Ewing sarcoma Nat Rev Dis Primers. 2018;4(1):5.

2. Gaspar N, Hawkins DS, Dirksen U, Lewis IJ, Ferrari S, Le Deley MC, et al. Ewing Sarcoma: Current Management and Future Approaches Through Collaboration. J Clin Oncol. 2015;33(27):3036-46.

3. Leavey PJ, Mascarenhas L, Marina N, Chen Z, Krailo M, Miser J, et al. Prognostic factors for patients with Ewing sarcoma (EWS) at first recurrence following multi-modality therapy: A report from the Children's Oncology Group. Pediatr Blood Cancer. 2008;51(3):334-8.

4. Stahl M, Ranft A, Paulussen M, Bölling T, Vieth V, Bielack S, et al. Risk of recurrence and survival after relapse in patients with Ewing sarcoma. Pediatr Blood Cancer. 2011;57(4):549-53.

5. Musa J, Cidre-Aranaz F, Aynaud MM, Orth MF, Knott MML, Mirabeau O, et al. Cooperation of cancer drivers with regulatory germline variants shapes clinical outcomes. Nat Commun. 2019;10(1):4128.

6. Aye Y, Li M, Long MJ, Weiss RS. Ribonucleotide reductase and cancer: biological mechanisms and targeted therapies. Oncogene. 2015;34(16):2011-21.

7. Goss KL, Gordon DJ. Gene expression signature based screening identifies ribonucleotide reductase as a candidate therapeutic target in Ewing sarcoma. Oncotarget. 2016;7(39):63003-19.

8. Goss KL, Koppenhafer SL, Harmoney KM, Terry WW, Gordon DJ. Inhibition of CHK1 sensitizes Ewing sarcoma cells to the ribonucleotide reductase inhibitor gemcitabine. Oncotarget. 2017;8(50):87016-32.

9. Finch RA, Liu M, Grill SP, Rose WC, Loomis R, Vasquez KM, et al. Triapine (3-aminopyridine-2-carboxaldehyde- thiosemicarbazone): A potent inhibitor of ribonucleotide reductase activity with broad spectrum antitumor activity. Biochem Pharmacol. 2000;59(8):983-91.

10. Oesterheld JE, Reed DR, Setty BA, Isakoff MS, Thompson P, Yin H, et al. Phase II trial of gemcitabine and nab-paclitaxel in patients with recurrent Ewing sarcoma: A report from the National Pediatric Cancer Foundation. Pediatr Blood Cancer. 2020;67(7):e28370

11. Chao J, Synold TW, Morgan RJ Jr, Kunos C, Longmate J, Lenz HJ, et al. A phase I and pharmacokinetic study of oral 3-aminopyridine-2-carboxaldehyde thiosemicarbazone (3-AP, NSC \#663249) in the treatment of advanced-stage solid cancers: a California Cancer Consortium Study. Cancer Chemother Pharmacol. 2012;69(3):835-43.

12. Wadler S, Makower D, Clairmont C, Lambert P, Fehn K, Sznol M. Phase I and pharmacokinetic study of the ribonucleotide reductase inhibitor, 3-aminopyridine-2-carboxaldehyde thiosemicarbazone, administered by 96-hour intravenous continuous infusion. J Clin Oncol. 2004;22(9):1553-63.
13. Wang J, Lohman GJ, Stubbe J. Enhanced subunit interactions with gemcitabine-5'-diphosphate inhibit ribonucleotide reductases. Proc Natl Acad Sci U S A. 2007:104(36):14324-9.

14. Zhou B, Su L, Hu S, Hu W, Yip ML, Wu J, et al. A small-molecule blocking ribonucleotide reductase holoenzyme formation inhibits cancer cell growth and overcomes drug resistance. Cancer Res. 2013;73(21):6484-93.

15. Kunos CA, Radivoyevitch T, Ingalls ST, Hoppel CL. Management of 3-aminopyridine-2-carboxaldehyde thiosemicarbazone-induced methemoglobinemia. Future Oncol. 2012;8(2):145-50.

16. Stadler WM, Desai AA, Quinn DI, Bukowski R, Poiesz B, Kardinal CG, et al. A Phase I/II study of GTI-2040 and capecitabine in patients with renal cell carcinoma. Cancer Chemother Pharmacol. 2008;61(4):689-94.

17. Leighl NB, Laurie SA, Chen XE, Ellis P, Shepherd FA, Knox JJ, et al. A phase I/II study of GTI-2040 plus docetaxel as second-line treatment in advanced non-small cell lung cancer: a study of the PMH phase II consortium. J Thorac Oncol. 2009;4(9):1163-9.

18. Sridhar SS, Canil CM, Chi KN, Hotte SJ, Ernst S, Wang L, et al. A phase II study of the antisense oligonucleotide GTI-2040 plus docetaxel and prednisone as first-line treatment in castration-resistant prostate cancer. Cancer Chemother Pharmacol. 2011:67(4):927-33.

19. Barker CA, Burgan WE, Carter DJ, Cerna D, Gius D, Hollingshead MG, et al. In vitro and in vivo radiosensitization induced by the ribonucleotide reductase inhibitor Triapine (3-aminopyridine-2-carboxaldehyde-thiosemicarbazone). Clin Cancer Res. 2006;12(9):2912-8.

20. Dai L, Lin Z, Qiao J, Chen Y, Flemington EK, Qin Z. Ribonucleotide reductase represents a novel therapeutic target in primary effusion lymphoma. Oncogene. 2017;36(35):5068-74.

21. Oo ZY, Proctor M, Stevenson AJ, Nazareth D, Fernando M, Daignault $\mathrm{SM}$, et al. Combined use of subclinical hydroxyurea and CHK1 inhibitor effectively controls melanoma and lung cancer progression, with reduced normal tissue toxicity compared to gemcitabine. Mol Oncol. 2019;13(7):1503-18.

\section{Publisher's Note}

Springer Nature remains neutral with regard to jurisdictional claims in published maps and institutional affiliations.
Ready to submit your research? Choose BMC and benefit from:

- fast, convenient online submission

- thorough peer review by experienced researchers in your field

- rapid publication on acceptance

- support for research data, including large and complex data types

- gold Open Access which fosters wider collaboration and increased citations

- maximum visibility for your research: over 100M website views per year

At BMC, research is always in progress.

Learn more biomedcentral.com/submissions 